Pensamiento Crítico No. 7, pág. 103 - 110

\title{
Uso de las Investigaciones de Mercado por los Pequeños Empresarios
}

\author{
José Francisco Pinto Castro
}

Resumen: En el presente artículo se trata de resaltar la necesidad de usar las investigaciones de mercado para superar los problemas de falta de competitividad de las pequeñas empresas.

Palabras Claves: Investigación transversal, Investigación longitudinal, Entrevista en profundidad, Sesiones de grupo, Focus group, Entrevistas directas, encuestas, Panel de consumidores, Auditoria de negocios.

\section{Introducción y Antecedentes}

Según PROMPYME, "El número total de MYPE formales a noviembre del 2004 ascendió a 648,147 , lo cual representa el $98.35 \%$ del universo empresarial formal nacional" por lo que se ha convertido en el más importante del país. 
Cuadro 1 Perú:

Distribución de las empresas formales según rango de tamaño (a noviembre del 2004)

\begin{tabular}{|lrr|}
\hline Tipo de empresa & Número de empresas & Porcentaje \\
\hline Microempresa 1/ & 622,209 & $94.41 \%$ \\
\hline Pequeña empresa 2/ & 25,938 & $3.94 \%$ \\
\hline Mediana y Gran empresa 3/ & 10,899 & $1.65 \%$ \\
\hline Total & 659,046 & $100.00 \%$ \\
\hline
\end{tabular}

1/Ventas anuales brutas menores o iguales a 150 UIT. 2/Aproximación. Ventas brutas anuales menores o iguales a 850 UIT

3/ Aproximación. Ventas brutas anuales mayores a 850 UIT. Fuente: SUNAT 2005.

Elaboración: Dirección Nacional de la Micro y Pequeña Empresa.

En este mismo trabajo se cita que durante el 2004, "el $81 \%$ de las microempresas vendió menos de US\$1,100 mensuales, con un nivel de ventas promedio de US\$ 538, el 16\% de las microempresas, cuyo rango de ventas mensuales varía entre los US $\$ 1,100$ y US $\$ 6,300$, vendió en promedio US $\$ 2,622$, y que sólo un $3 \%$ obtuvo ventas mensuales entre US\$ 6,300 y US\$ 12,660 , con ventas promedio de US\$8,830. En cuanto a las pequeñas empresas, el nivel de ventas mensual promedio ascendió a US $\$ 26,773$, contribuyendo con el $49.6 \%$ del volumen de ventas de las MYPE, a pesar de que sólo representan el $4 \%$ de este estrato empresarial".

En lo que respecta a la presencia de las MYPE en los diferentes sectores económicos, según este mismo trabajo de investigación, "la MYPE representa el 99 \% del total de empresas formales en cada uno de los sectores comercio y servicios".

Por otro lado, en este mismo trabajo se cita a Fernando Villarán quien dice: "A pesar de la trascendencia de la MYPE en términos numéricos y de su absorción de la PEA ocupada, su contribución al Producto Bruto Interno (PBI) sólo alcanza al 42.1\%”.

Como se puede ver en el Cuadro $\mathrm{N}^{\circ}$ 2, la mayor contribución de la MYPE al PBI (21.2\%) se concentra en el sector servicios, seguido de los sectores agricultura, (10.4\%), manufactura (6.9\%), construcción (3.1\%), pesca $(0.3 \%$ ) y minería $(0.1 \%)$.

\section{Cuadro 2. Participación de las MYPE en el PBI}

\begin{tabular}{|l|rrr|}
\hline Sector Económico & $\begin{array}{r}\text { Participación } \\
\text { Sectorial /1 }\end{array}$ & $\begin{array}{r}\text { MYPE en cada } \\
\text { sector /2 }\end{array}$ & $\begin{array}{r}\text { Participación } \\
\text { de MYPE /3 }\end{array}$ \\
\hline Agricultura & 11.6 & 90.0 & 10.4 \\
\hline Pesca & 1.0 & 30.0 & 0.3 \\
\hline Minería & 2.5 & 5.0 & 0.1 \\
\hline Manufactura & 23.1 & 30.0 & 6.9 \\
\hline Construcción & 8.8 & 35.0 & 3.1 \\
\hline Servicios & 53.0 & 40.0 & 21.2 \\
\hline Total & $\mathbf{1 0 0 . 0}$ & & $\mathbf{4 2 . 1}$ \\
\hline
\end{tabular}

1/ Contribución de cada sector en el PBI. Fuente INEI 3/ Participación global de la MYPE en el PBI $(3=1 \times 2)$

2/ Participación de la MYPE en el producto de cada sector 


\section{Uso de las investigaciones de mercado por los pequeños empresarios}

Por otro lado, las empresas que más quiebran son las MYPE debido a sus restricciones internas: baja productividad, baja competitividad y baja rentabilidad y debido a sus restricciones externas. ${ }^{4}$

Actualmente las economías atraviesan un proceso de globalización que implica un desarrollo de las tecnologías de la información y comunicación lo que ha traído como consecuencia, la creciente dificultad de las MYPE para competir en los mercados debido a, entre otras cosas, a problemas de información.

En un mundo tan competitivo como el de ahora, las pequeñas empresas tienen que enfrentarse a las grandes empresas en desigualdad de condiciones debido a la falta de recursos y principalmente, a la falta de información.

Las grandes empresas cuentan con personal más capacitado y mejor informado, que sabe de la importancia de tener información actualizada. Por eso, pueden aprovechar mejor las oportunidades y resolver los problemas de su empresa, de forma más rápida.

La mayoría de empresas peruanas tienen que enfrentar a las empresas extranjeras con esta desventaja porque sencillamente no le dan el valor que debe a las investigaciones de mercado y esto principalmente por la falta de dinero para pagar una investigación de este tipo y la excesiva confianza en su propia experiencia.

Muchas empresas de nuestro país no cuentan con un sistema de información. Solo recurren a la búsqueda de información en circunstancias extremas. Es decir, cuando tienen que tomar decisiones muy importantes, que implican un alto nivel de inversión.

Las empresas de investigación de mercados, suelen ofrecer sus informes principalmente a las grandes empresas. No han pensado en vender sus servicios a la pequeña empresa porque saben que ellas no están dispuestas a desembolsar parte de sus ingresos en este tipo de trabajos y que más que todo se basan en su experiencia, para atender el mercado.

Los diseños de investigación que ofrecen las empresas de investigación de mercados los podemos clasificar de diversas formas. Tomando en cuenta el grado de control de las variables, se pueden clasificar en: experimentales, cuasi experimentales y observacionales.

Tambien se pueden clasificar de acuerdo a su direccionalidad (prospectiva, retrospectiva y bidireccional) o tomando en cuenta el número de ocasiones en el que se evalúan las variables (transversales y longitudinales) y de acuerdo al número de muestras que se estudian (descriptivos y comparativos). ${ }^{5}$ 


\section{José Francisco Pinto Castro}

En el presente artículo, evaluaremos los diseños de investigación clasificados por el número de ocasiones en el que se realiza la medición de las variables en estudio. Estos se clasifican en transversales y longitudinales. Los transversales son aquellas investigaciones que estudian un grupo en un momento y lugar determinado, pero admitiendo que existen subgrupos con el fin de estudiarlos a la vez con respecto a un grupo de variables importantes para el investigador. Mientras que los longitudinales consisten en hacer dos o más estudios del mismo grupo en tiempos diversos. ${ }^{6}$

Por otro lado, la mayoría de empresas de investigación de mercados peruanas ofrecen los siguientes tipos de investigación: entrevistas en profundidad, sesiones de grupo (focus group), entrevistas (encuestas), paneles de consumidores y auditorias de negocios.

Las entrevistas en profundidad son investigaciones cualitativas que se emplean normalmente cuando no se tiene la posibilidad de conseguir con facilidad la información de las personas que pertenecen al mercado objetivo. Kinnear y Taylor definen las entrevistas en profundidad como "una entrevista personal no estructurada que utiliza una indagación exhaustiva para lograr que un solo encuestado hable libremente y exprese en forma detallada sus persuasiones y sentimientos sobre el tema"?

La ventaja de este tipo de investigación está en que puede obtener información mas precisa sobre las causas que motivan el comportamiento de los entrevistados. Pero un gran inconveniente se puede relacionar con el sesgo que pueden ocasionar los entrevistados por pertenecer a un cierto segmento de mercado y la completa dependencia respecto al entrevistador para el análisis y la interpretación de la información.

Las sesiones de grupo son otro tipo de investigación cualitativa que se diferencia de las entrevistas en profundidad por recoger los datos de los participantes en forma grupal.

Según Zikmund, una sesión de grupo "es una entrevista libre y no estructurada que se realiza a un pequeño grupo de personas".

Se considera que este tipo de investigación tiene una ventaja sobre las entrevistas en profundidad porque consideran a más participantes y la interacción de más personas da confianza a los participantes para expresar sus opiniones libremente. Sus desventajas se relacionan con el moderador, que debe ser sensible y eficaz, y con la forma en que se escoge a los participantes, que en algunas ocasiones genera dificultades por no haber sido elegidos de forma correcta.

Las entrevistas, que son investigaciones que se emplean para desarrollar investigaciones del tipo cuantitativo, se pueden clasificar en entrevistas por correo, entrevistas telefónicas, 


\section{Uso de las investigaciones de mercado por los pequeños empresarios}

entrevistas personales y paneles.

Actualmente, el desarrollo de la tecnología permite aplicar entrevistas por correo electrónico o empleando una página Web.

La más usada de todas estas es la entrevista personal. Según Fisher y Navarro, "es uno de los procedimientos más utilizados, ya que permite obtener mejores resultados"

La ventaja de este tipo de investigación se relaciona con la proyección de los resultados obtenidos de un grupo representativo a todo el mercado objetivo pues consideran una formula estadística que le da validez a los resultados obtenidos. El número de participantes se obtiene por medio de una formula estadística que toman en cuenta el tamaño de la población, un nivel de confianza, un margen de error, una probabilidad a favor y una probabilidad en contra de que ocurra un determinado acontecimiento.

Su desventaja está en los problemas que podrían surgir en relación al costo de llevarla a cabo y en relación con la cantidad de entrevistadores que participan, pues a mayor número de entrevistadores existe una mayor probabilidad de cometer errores en el momento de realizar las entrevistas.

"Los paneles son muestras de encuestas que proporcionan información específica a intervalos regulares por un periodo extenso" 10 Este periodo puede ser de seis a doce meses.

Los paneles sirven para pronosticar las ventas, estimar la participación de mercado, evaluar la lealtad a la marca y la conducta de sustitución de marcas, establecer perfiles de usuarios específicos y medir la eficacia de las promociones.

Una ventaja de este tipo de investigación es la mayor predisposición de la gente que participa por lo que se pueden obtener datos de mayor calidad que los obtenidos en una encuesta. Además del hecho de que la información se anota justo en el momento de la compra con lo que se eliminan los errores de la memoria.

Una gran desventaja de este tipo de investigación se relaciona con la falta de representatividad que pueden tener los participantes, pues en algunas ocasiones no se incluyen a todos los segmentos de mercado que deben estar incluidos. Además, tambien se puede presentar como un problema importante el cansancio de los participantes que puede ocasionar desgano y falta de colaboración y la aparición de respuestas desviadas.

Por otro lado, "las auditorias son un examen y verificación formal del movimiento de un producto".1. Se realizan en los negocios minoristas y mayoristas. Los que participan reciben 


\section{José Francisco Pinto Castro}

información básica y pagos en efectivo por el servicio de auditoria.

Los datos de la auditoria se enfocan en los productos o servicios vendidos a través de las tiendas o las características de las tiendas mismas.

Este tipo de investigación se usa por lo general para determinar el tamaño del mercado total y la distribución de ventas por tipo de tienda, región o ciudad, evaluar la participación de marcas y la actividad competitiva, identificar la asignación del espacio de anaquel y problemas de inventario, analizar los problemas de distribución, establecer los potenciales de ventas y pronósticos y establecer y vigilar las asignaciones promociónales.

Las auditorias sirven para proporcionar información relativamente exacta acerca del movimiento de diversos productos a niveles de mayoristas y minoristas. Además, este tipo de información se puede dividir en un importante número de variables. Sin embargo, las auditorias tienen cobertura limitada. Asimismo puede no ser oportuna o actual. Además, a diferencia de los datos que proporcionan los lectores ópticos, los datos de auditoria no se pueden ligar a las características del consumidor.

En nuestro mercado, el costo de tener información actualizada es muy alto y es por ese motivo que las empresas peruanas y principalmente las pequeñas empresas no son capaces de acceder a este tipo de información.

Es necesario señalar que el precio de una investigación de mercados depende del tipo de investigación y del prestigio de la empresa que va hacer la investigación. Así tenemos que una investigación transversal del tipo cualitativo puede costar entre US $\$ 2100$ y US $\$ 3100$ mientras que las entrevistas personales (encuestas) pueden llegar a costar entre US $\$ 2,500$ y US $\$ 10,000$, dependiendo de la complejidad con que se puede captar a los participantes.

Al respecto, hemos encontrado una tabla referencial de precios de los focus group o sesiones de grupo el cual, a continuación transcribimos.12

\section{Cuadro: Precios de Sesiones de Grupos}

\begin{tabular}{|l|r|}
\hline Institución & Precio \\
\hline Apoyo & US\$ 1,000 \\
\hline Conecta & US\$700 \\
\hline Mercadeando & US\$ 350 \\
\hline Mercadeando* & US\$ 45 \\
\hline
\end{tabular}

* Precio por hora para asesoría en desarrollo de productos. 
Los precios mostrados en el cuadro de arriba son por la realización de una sesión de grupo recomendándose hacer entre cuatro y seis sesiones de grupo.

En realidad, no basta con hacer una investigación para ser competitivo. Se necesita tener información constante del mercado para tomar en cuenta los cambios que se dan en los gustos y preferencias del consumidor y enfrentar a los competidores con mejores productos $\mathrm{y} / \mathrm{o}$ servicios.

Por este motivo, se hace necesario que una empresa desarrolle constantes investigaciones sobre su mercado objetivo y no solo empleen las investigaciones de tipo transversal sino además, las investigaciones de tipo longitudinal. Las investigaciones que se agrupan bajo este nombre son los paneles y las auditorias.

El costo de una investigación de mercados del tipo longitudinal (paneles y auditorias), está prácticamente fuera del alcance de las medianas y pequeñas empresas. Normalmente, estas investigaciones se venden en forma anual y su precio está por arriba de los US $\$ 50,000$.

Estos tipos de investigación son los que más sirven y a los que más usualmente recurren las grandes empresas por ser la más exacta para tomar decisiones.

Es necesario poner al alcance de la pequeña empresa las investigaciones de mercado. Sería la mejor forma de conseguir que las pequeñas empresas se vuelvan competitivas en un menor tiempo.

No se pueden desarrollar productos sin conocer las preferencias de los consumidores ni sin conocer lo que la competencia está haciendo para que sean sus clientes.

Por eso, resulta importante conocer la disposición que podrían tener los pequeños empresarios respecto al uso de información de mercado a un costo reducido.

Se necesita averiguar si, una vez que se elimina el problema de los costos, los pequeños empresarios pueden usar esta información sin problema y volverse competitivos al enfrentar a las grandes empresas.

\section{Bibliografía}

Malhotra, Naresh K. "Investigación de mercados: Un Enfoque aplicado", 4ta Edición. Pearson Educación, México, 2004. 


\section{José Francisco Pinto Castro}

Zikmund, William G. "Investigación de Mercados". 6ta Edición. Editorial Prentice Hall Hispanoamericana. México. 1997

Fisher, Laura y Navarro Alma. "Introducción a la Investigación de Mercados", 3ra Edición. Editorial McGraw Hill Interamericana Editores S.A. de C.V. 1996

Kinnear, Thomas C. y Taylor, James R. "Investigación de Mercados: Un enfoque aplicado". 4ta Edición. Editorial McGraw Hill. Bogota, Colombia. 1993

\section{Notas Referenciales}

1. "La situación de la Micro y Pequeña Empresa en el Perú", Ministerio de Trabajo y Promoción del Empleo, PROMPYME y Gobierno del Perú. Junio 2005

2. Ibid

3. Ibid

4. "Modelos de Economía Solidaria en el Perú: El Caso de la Micro y Pequeña Empresa" - Corina Quintana Rodríguez. Oficina de Servicios Empresariales", Ministerio de Trabajo y Promoción del Empleo, PROMPYME y Gobierno del Perú. Diciembre, 2005

5. Óscar C Thompson Chagoyán. "Diseños de investigación en ciencias de la salud”. En: Rev Hosp Gral Dr. M Gea González, Vol 3, No. 4, Octubre-Diciembre 2000, Págs. 182-186, http://medigraphic.com/pdfs/h-gea/gg2000/gg004h.pdf Miércoles 20 de Junio de 2007, 8:00 horas

6. "Trabajo Práctico, Tema: Diseños de Investigación". En: Introducción a la Biología. Departamento de Biología. FACENA-UNNE, http://exa.unne.edu.ar/depar/areas/biologia/introbio/public_html/2.pdf. Miércoles 20 de Junio de 2007, 8:10 horas

7. Kinnear, Thomas y Taylor, James R. "Investigación de Mercados: Un enfoque aplicado". 4ta Edición. McGraw Hill. Bogota, Colombia. 1993

8. Zikmund, William G. "Investigación de Mercados". 6ta Edición. Editorial Prentice Hall Hispanoamericana. México. 1997

9. Fisher, Laura y Navarro Alma. "Introducción a la Investigación de Mercados", 3ra Edición. Editorial McGraw Hill Interamericana Editores S.A. de C.V. 1996

10. Malhotra, Naresh K. "Investigación de mercados: Un Enfoque aplicado", 4ta Edición. Pearson Educación, México, 2004

11. Malhotra, Naresh K., ibid.

12. Suplemento Mi Negocio del diario El Comercio del 17 de junio del 2007 , página 3. 